\title{
Tension gastrothorax: a life-threatening cause of acute abdominal pain
}

\author{
Yuan Kao MD, Wei-Jing Lee MD, Hung-Jung Lin MD
}

$\mathrm{A}$ n otherwise healthy 52-year-old man was admitted to hospital following a motor vehicle crash in which he had suffered a contusion on the left side of his chest, multiple rib fractures and a hemothorax. After 18 days of conservative management, which included drainage with a chest tube, he was discharged home. A year later, he presented to the emergency department with a 2-day history of abdominal fullness, epigastric pain and intractable hiccups. An examination revealed severe epigastric tenderness, facial cyanosis and engorgement of the jugular vein. A chest radiograph showed a large cavitary mass with an air-fluid level in the left side of the hemithorax and a shift of the mediastinum to the right (Figure 1). We were unable to insert a nasogastric tube. The tract seemed to be obstructed, possibly because of tension gastrothorax. Our patient became agitated and cyanotic, and, within a few minutes, cardiac arrest occurred. Following resuscitation and stabilization of his vital signs, an emergent thoracotomy was performed, and an $8-\mathrm{cm}$ diaphragmatic laceration was repaired. The patient died 24 days later in hospital from complications, which included sepsis.

Tension gastrothorax is associated with congenital diaphragmatic defects and certain surgical procedures, including chest-tube thoracostomy, laparoscopic cholecystectomy, microwave-assisted laparoscopic hepatectomy, laparoscopic gastric banding, and plication for eventration of the diaphragm. Tension gastrothorax caused by a traumatic rupture of the diaphragm is rare and difficult to diagnose. ${ }^{1}$ The incidence of diaphragmatic injury among patients with blunt thoracic and abdominal trauma is about $3 \%-5 \% .^{2}$ The incidence increases to $10 \%-15 \%$ if there has been a traumatic chest penetration. ${ }^{2}$ Following blunt trauma, injuries to the left side of the diaphram are 3 times more common than injuries to the right side, likely a result of the liver's buffering effect. ${ }^{3}$

The definitive treatment for tension gastrothorax is operative repair, but emergent decompression with a nasogastric tube, possibly aided by endoscopy, can help control the situation. Emergency nasogastric tube placement can be technically challenging because of kinking at the level of the diaphragm and may lead to further hyperventilation and gastric distension if incorrectly performed. ${ }^{4}$ Awareness of the risk factors and the clinical presentation of tension gastrothorax can help with the prompt identification and successful management of this life-threatening condition.

From the Department of Emergency Medicine, Chi-Mei Medical Center, Yung-Kang City, Taiwan

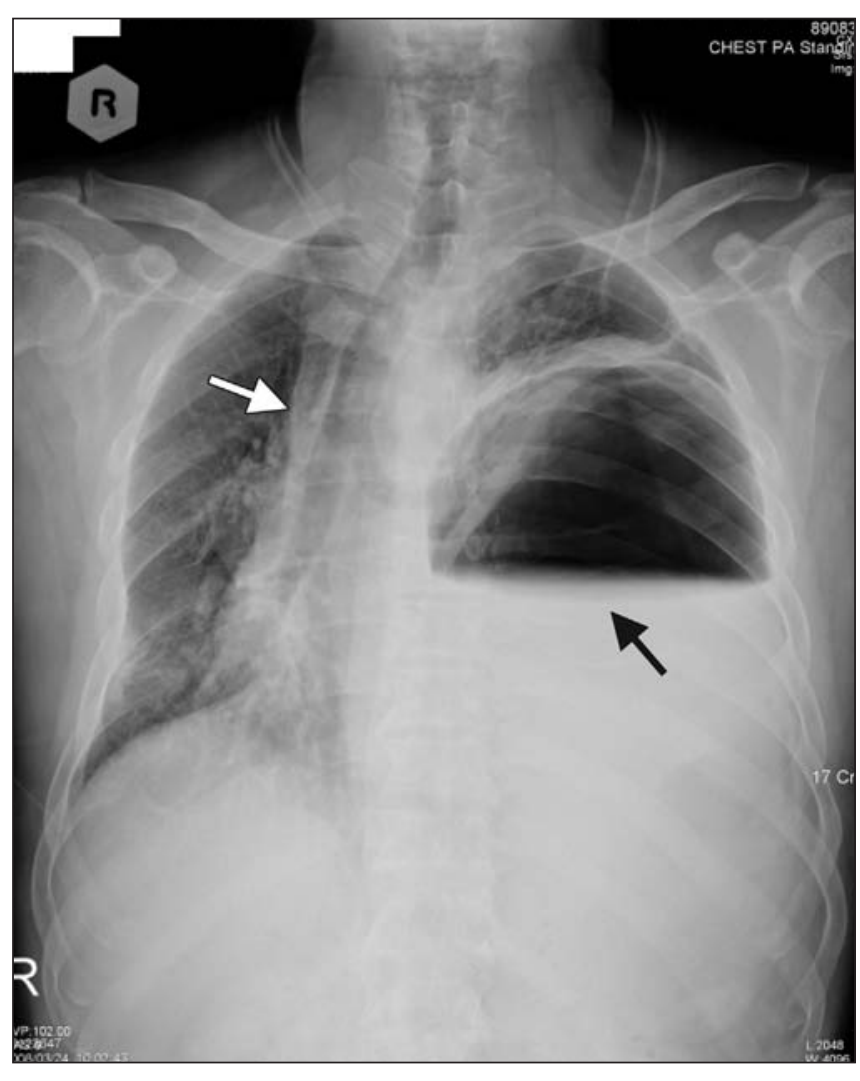

Figure 1: A radiograph of the patient's chest showing a large cavitary mass in the left lung field with air-fluid level (black arrow) and a shift of the mediastinum to the right (white arrow).

\section{REFERENCES}

1. Lee WJ, Lee YS. Traumatic diaphragmatic rupture: a diagnostic challenge in the emergency department. Emerg Med J 2007;24:601.

2. Nishijima D, Zehbtachi S, Austin RB. Acute posttraumatic tension gastrothorax mimicking acute tension pneumothorax. Am J Emerg Med 2007;25:734.e5-6.

3. Iochum S, Ludig T, Walter F, et al. Imaging of diaphragmatic injury: A diagnostic challenge? Radiographics 2002 Oct;22 Spec No:S103-16; discussion S116-8.

4. Bamgbade OA. Management of tension gastrothorax. Resuscitation 2006;70:293-4.

Clinical images are chosen because they are particularly intriguing, classic or dramatic. Submissions of clear, appropriately labelled high-resolution images must be accompanied by a figure caption and the patient's written consent for publication. A brief explanation (300 words maximum) of the educational significance of the images with minimal references is required. 